4 discharge from hospital or the end of the study (31 March 2016).

Of these patients, $16 \%$ received preoperative oral care from a dentist. When a surgeon requested that a dentist provide preoperative oral care to a patient with cancer, the dentist checked the patient's oral condition, provided professional tooth cleaning, taught the patient self-cleaning methods for the teeth, and provided any treatment needed.

Analysis of the data showed that 15,724 patients (3.09\%) developed postoperative pneumonia and $1,734(0.34 \%)$ died within 30 days of surgery.

After adjustments, preoperative oral care by a dentist was linked with a decrease in postoperative pneumonia from $3.76 \%$ to
$3.28 \%$ and death within 30 days from $0.42 \%$ to $0.30 \%$.

The authors said: 'The findings could help improve strategies for the prevention of postoperative complications.'

1. Ishimaru M, Matsui $H$, Ono S, Hagiwara $Y$, Morita $\mathrm{K}$, Yasunaga $\mathrm{H}$. Preoperative oral care and effect on postoperative complications after major cancer surgery. Br J Surg 2018; DOI:10.1002/bjs.10915. (accessed on 14 August 2018).

\title{
Largest oral HPV study in England shows lower than expected infection rates
}

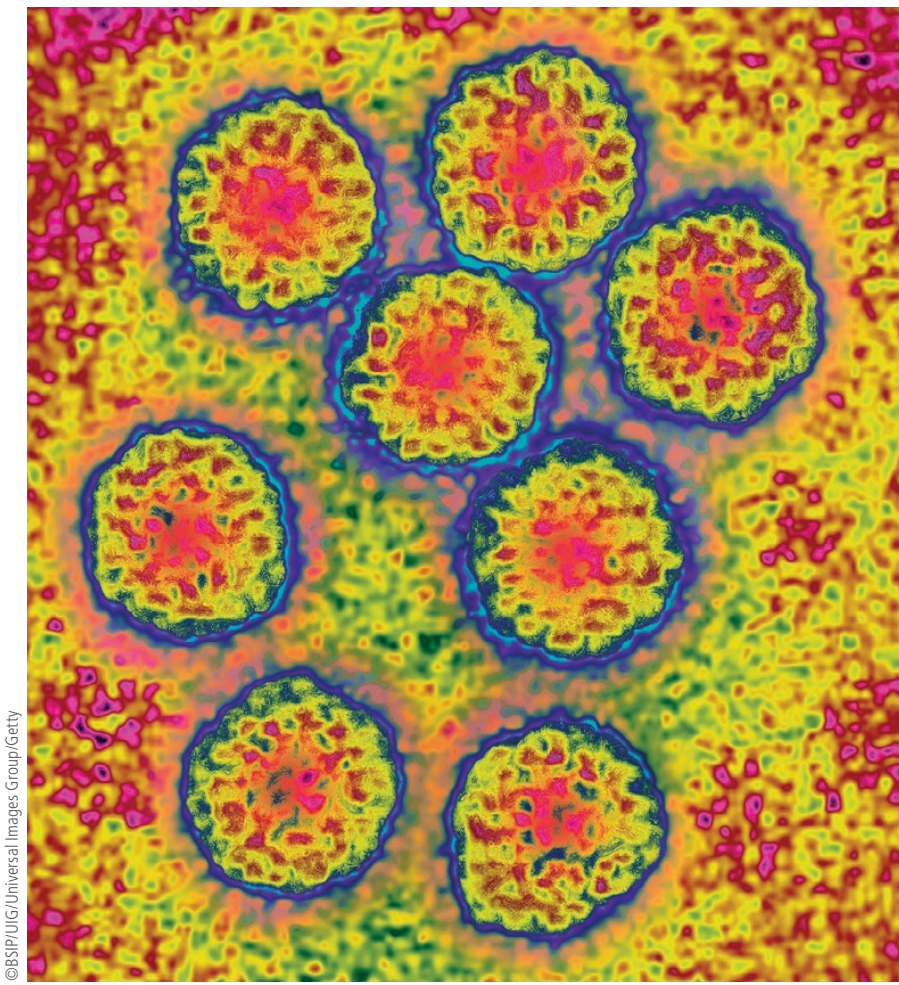

The largest study so far of high risk human papillomavirus (HR-HPV) oral infection in England has shown that infection rates are lower than expected, compared to previous studies.

The research, ${ }^{1}$ conducted by the University of Sheffield, also showed that smoking and sexual behaviour were risk factors for oral HPV infection, which can lead to oropharyngeal (throat) cancer.

The study was published in the online journal BMJ Open on 19 August 2018 and was led by the University's Professor Hilary Powers, Dr Vanessa Hearnden and Dr Craig Murdoch and funded by the World Cancer Research Fund UK.

It coincides with the announcement of a new UK HPV vaccine programme for boys which will reduce the risk of HR-HPV related cancers.

Rates of oropharyngeal cancer (OPC) are rising worldwide, attributable to an increase in the incidence of oral infection with HR-HPV.

For the new study - described as the largest of its kind in England - researchers recruited 700 men and women in Sheffield and looked for HR-HPV infection and also asked participants lifestyle questions relating to their sexual history and tobacco use over the period of April 2013 to August 2014.

Analysis of the results showed that $2.2 \%$ of people were infected with oral HR-HPV infection with 0.7\% positive for HPV 16 or HPV 18.

There are large variations in oral HR-HPV prevalence globally, but this study showed lower rates compared to previous US ${ }^{2}$ and Scottish ${ }^{3}$ studies which both found $3.7 \%$ of individuals positive for oral HR-HPV.

Former smokers were significantly more likely to be HR-HPV positive compared with those that had never smoked and the study also found that participants with a greater number of sexual or oral sexual partners were more likely to be HR-HPV positive.

Dr Vanessa Hearnden, from the Department of Materials Science and Engineering at the University of Sheffield, said: 'Previous studies have been US-focused or in smaller UK studies in London or Scotland. This is the first study in the North of England and found lower rates of oral high-risk human papillomavirus infection.

'We fully support the newly announced HPV vaccination programme for boys which will reduce the risk of HPV-related cancers including throat cancer in men and will also provide further prevention of cervical cancers through herd immunity.

'However, we found the majority of individuals testing positive for high risk strains of HPV were actually positive for strains other than those covered by the current vaccine (HPV 16 and HPV 18). This shows the need to consider newer vaccines which protect against more HPV strains in the future and for individuals to be aware of lifestyle risk factors such as number of sexual partners and tobacco use.'

Dr Craig Murdoch, from the University's School of Clinical Dentistry, said: 'Many people associate the HPV virus with cervical cancer but there is less recognition of the fact that HPV causes oropharyngeal cancer, and unfortunately, the prevalence of this cancer has increased dramatically in the past few years.'

1. Hearnden V, Murdoch C, D'Apice K, Duthie S, Hayward N, and Powers H J. Oral human papillomavirus infection in England and associated risk factors: a case-control study. BM Open 2018; e022497. DOI:10.1136/bmjopen-2018-022497. http://dx.doi.org/10.1136/ bmjopen-2018-022497 (accessed on 20 August 2018).

2. Gillison M L, Broutian T, Pickard R K et al. Prevalence of oral HPV infection in the United States, 2009-2010. JAMA 2012; 307: 693-703. https://jamanetwork.com/journals/jama/ fullarticle/1104983. (accessed on 20 August 2018).

3. Conway D I, Robertson C, Gray H et al. Human Papilloma Virus (HPV) Oral Prevalence in Scotland (HOPSCOTCH): a feasibility study in dental settings, PLOS One 2016; 11: e0165847. http://journals.plos.org/plosone/article?id=10.1371/journal.pone.0165847. (accessed on 20 August 2018). 